\title{
O aparelho muscular dos Proglotes de Taenia Saginata
}

\author{
por \\ Antonio Augusto Xavier e Jonio F. de Salles
}

(Com cinco figuras no texto)

A documentação histológica apresentada neste trabalho refere-se exclusivamente a proglotes de Taenia saginata eliminados espontâneamente, cada um dêles representando, ccmo é óbvio, o último anel da cadeia constituindo o verme parasito.

Os proglotes foram fixados em formol a 10 p. 100 após 24 horas de permanência em baixa temperatura $\left(\mathrm{ca} .+20^{\circ} \mathrm{C}\right.$. $)$. Antes de serem submetidos a essa temperatura os proglotes mediam 35 milimetros de comprimento, e de largura 5 milimetros. Depois de permanecerem no refrigerador por 24 horas ou mais tempo, mostram-se êles retraídos em todos os seus diâmetros, medindo então de 8 a 12 milimetros de comprimento por 3 milimetros de diâmetro transverso, em sua maior largura.

Depcis de submetidos a baixa temperatura, como acima ja foi dito, os proglotes foram fixados em formol a 10 p. 100 durante 48 horas, sendo as peças depois transferidas para uma solução de formol a 4 p. 100 (solução ccnservadora), onde permaneceram por espaço de vários meses, para depois serem incluídas em parafina e cortadas. Para o estudo histológico foram utilizados os seguintes métodos de coloração: a) hematoxilina-eosina; $b$ ) azul de anilina de Mallory; c) tricrômico de Masson; d) impregnação pela prata segundo $\mathrm{Bi}$ elschowsky; e) impregnação pela prata segundo o método de Foot-Wilder.

O aparelho muscular dos proglotes de Taenia saginata dispõe-se em duas camadas distintas, uma delas, a mais externa, orientando-se no sentido do longo eixo do proglote, é a camada de músculo longitudinal; a outra camada, mais interna e em contato com o chamado parênquima do corpo, forma um envoltório de proteção aos órgãos internos, dispostas as suas fibras em sentido transversal. Uma outra camada de músculo circular dispõe-se imediatamente

* Recebido para publicação a 11 de dezembro de 1944 e dada à publicidade em fevereiro de 1945 . 


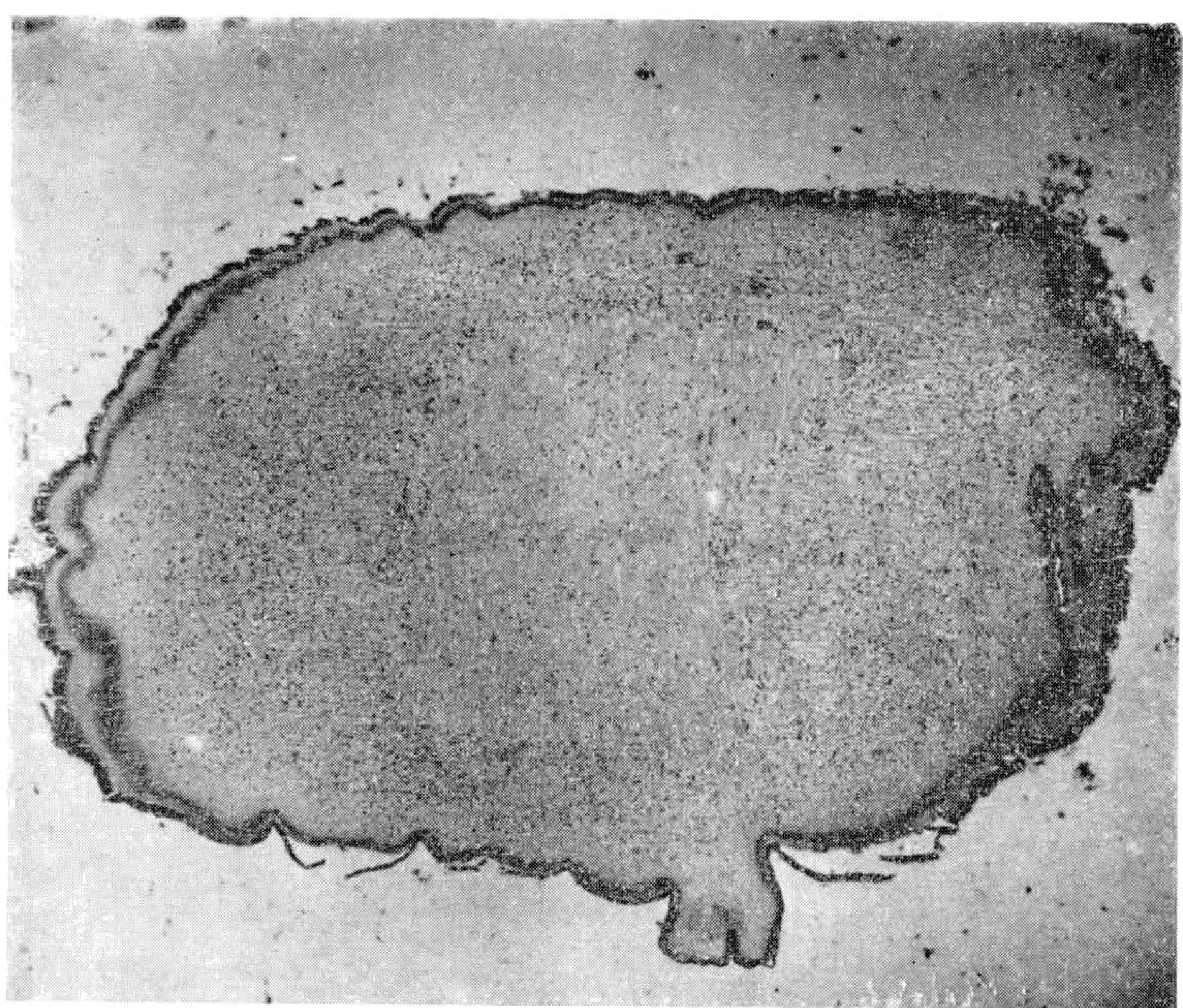

Fig. 1 - Córte histológico de um proglote. As fibras musculares dispõem-se em duas camadas distintas: a camada muscular longiturinal e a camada de músculo transversal. Col. Hematoxilina-eosina $\times 20$ - Mícrofoto $J$. Pinto.

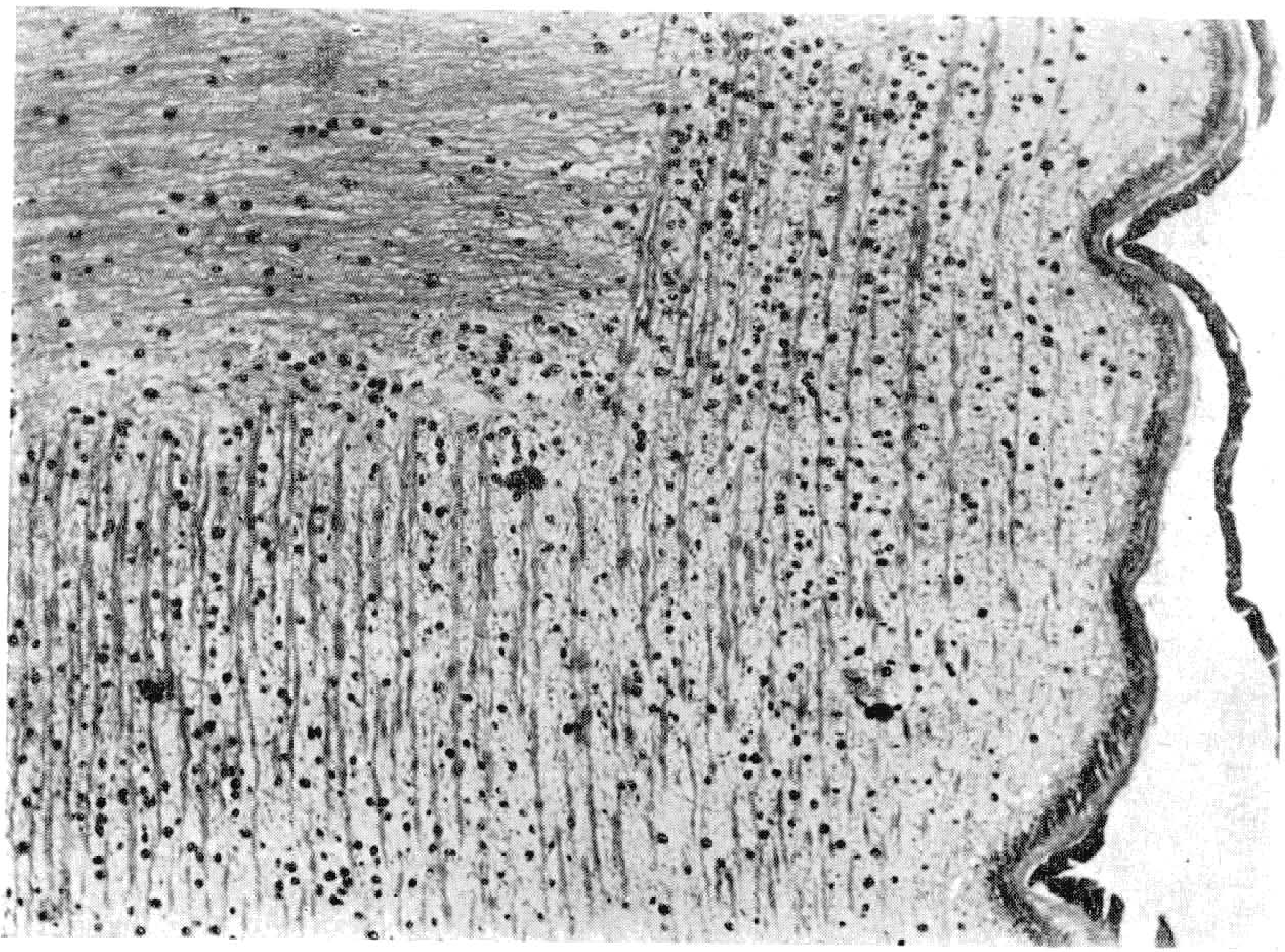

Fig. 2 - Vè-se nitidamente a disposicão das fibras musculares em duas camadas distintas Col. Hematoxilina-eosina $\times 130$ - Microfoto de $J$. Pinto. 


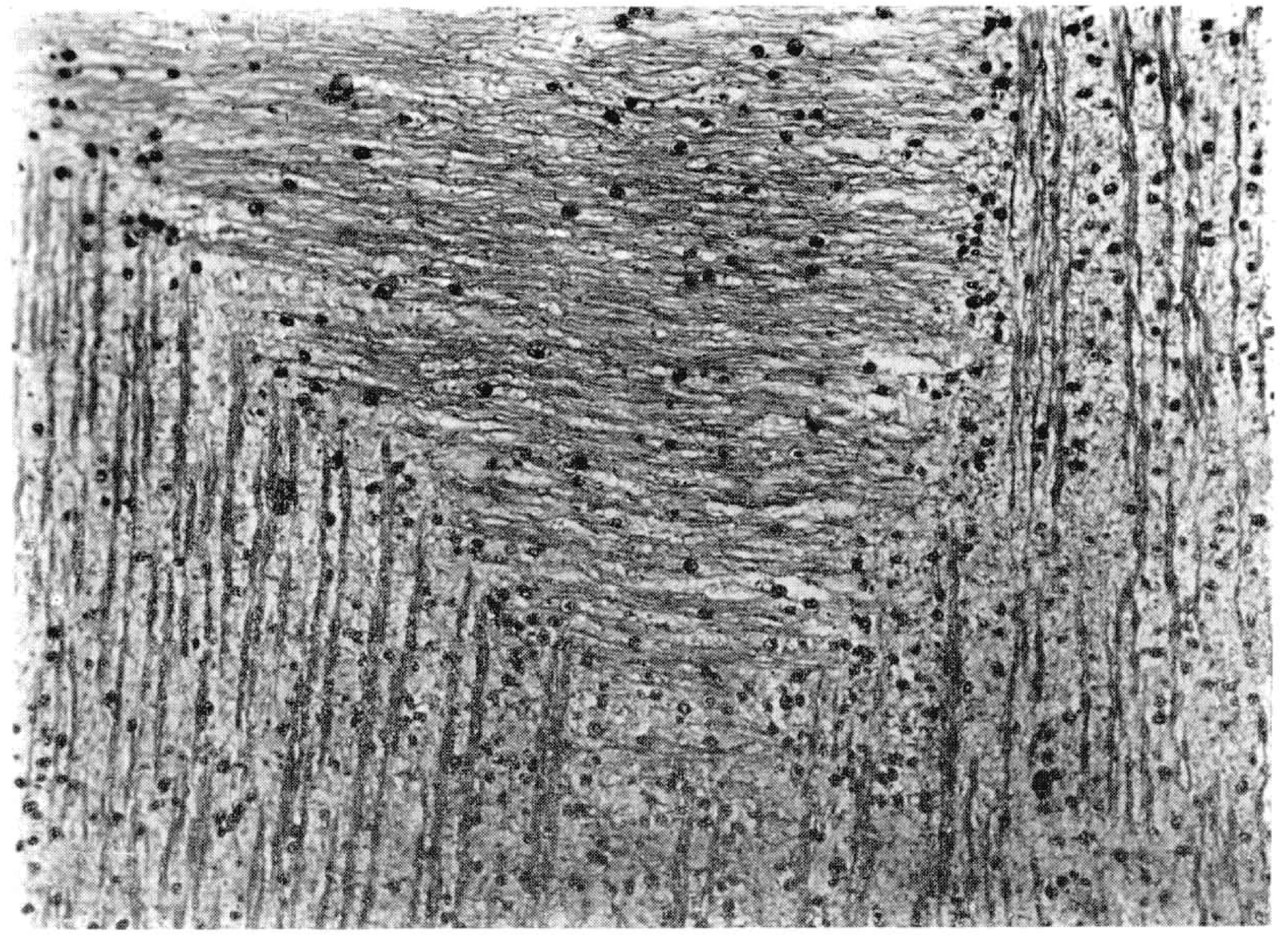

Fig. 3 - Fibras alongartas, possuindo nucléos pouco desenvolvidos excêntricos, dispostas longitudinalmente e fibras mais agrupadas, com núcleos Col. Hematoxilina-eosina $\times 130$ - Microfoto de $J$. Pinto.

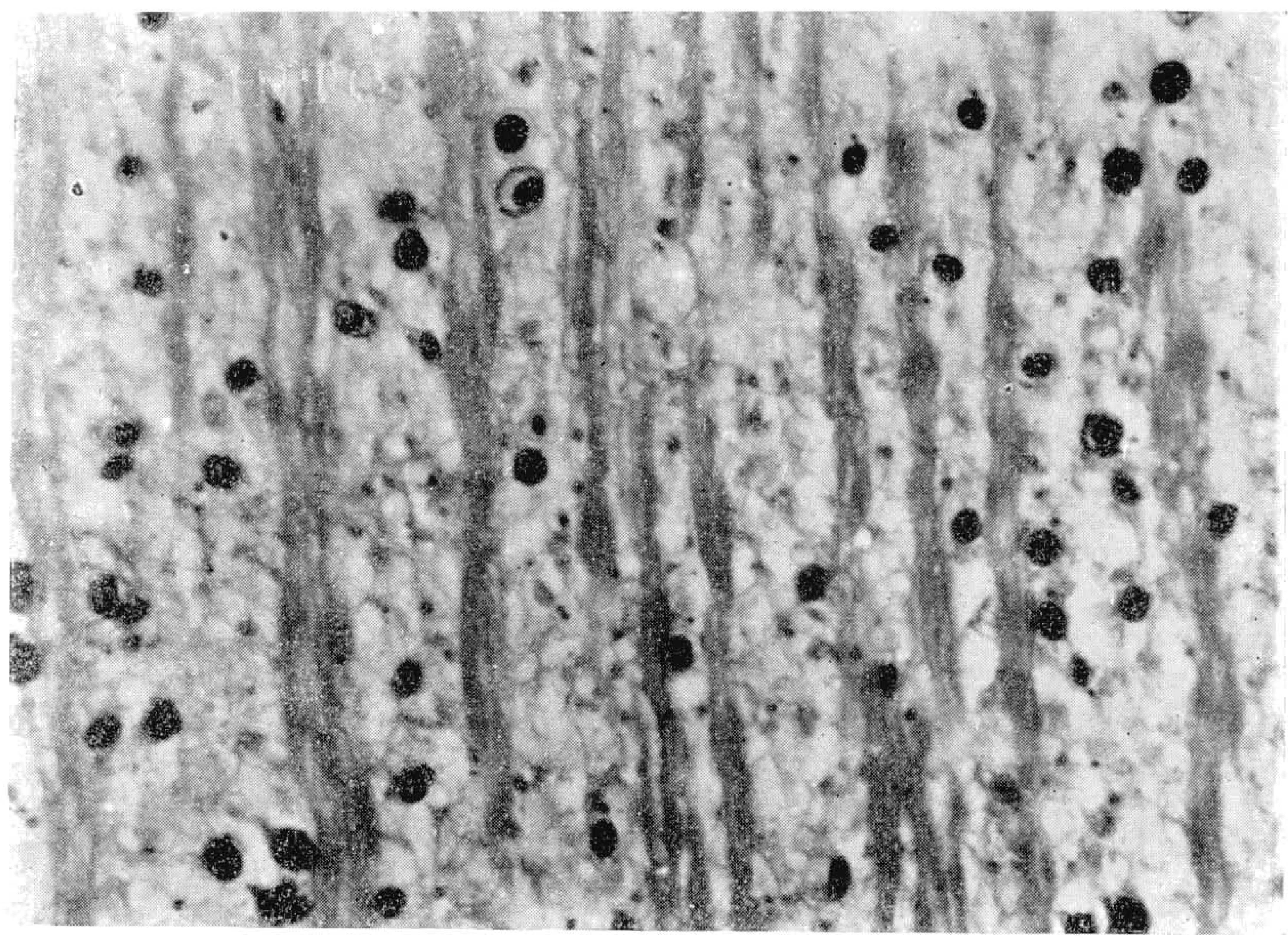


abaixo da cutícula. Devem ser assinaladas ainda as fibras dorso-ventrais unindo as paredes dorsal e ventral do corpo do proglote. Nossas preparações mostram tão sòmente o arranjo e a estrutura das fibras formando a camada muscular longitudinal e a camada de músculo transversal. As figuras 1, 2 e 3 demonstram a disposição das duas camadas musculares.



\footnotetext{
Fig. 5 - Fibras musculares dispostas em sentido longitudinal. Entre as fibras o fino retículo de fibrila impregnado pela prata, bem como, ovos do helminto Col. Foot-Wilder $\times 950$ - Mierofoto de $J$. Pinto.
}

As preparações histológicas mostram fibras musculares lisas, alongadas, dispostas longitudinalmente, exibindo núcleos pouco desenvolvidos, pálidos, em geral excêntricos; outras mais finas, orientadas em sentido transversal, cujos núcleos são pequenos e também excêntricos. (Figs. 3, 4 e 5). Um caráter interessante dos núcleos é que êles são sempre simples e excêntricos.

As fibras musculares lisas apresentam-se sempre anastomozadas, só por exceção encontrando-se fibras isoladas. Êste arranjo estrutural é, se possivel, 
ainda mais aparente nas extremidades marginais do proglote e em relação com a cuticula que reveste todo o tubo muscular, onde as fibras longitudinais se anastomozam ainda mais densamente, lembrando o todo o aspecto de uma cremalheira.

Entre as fibras musculares e em plano mais profundo, nota-se um tecido areolar de malhas irregulares, formado por finas trabéculas, onde se distinguem numerosos núcleos redondos das células do parênquina. (Figs. 4 e 5 ). E' possível observa-se aí a presença de corpos calcáreos incluídos nas malhas do retículo. (Fig. 5).

\section{THE MUSCLLAR TEXTURE OF THE PROGLOTTIDES OF TAENIA SAGINATA}

by

Antonio Augusto Xavier and Jonio F. de Salles

The writers describe the muscular texture of the proglottides of Taenia saginata, Goeze, 1782, based upon microscopical preparations of mature and gravid proglottides stained by several methods. The muscular system of the proglottides of Taenia saginata is disposed mainly in two layers, a longitudinal and external one, and a deeper transverse layer, lining the body parenchym and internal organs. A circular or annular layer ranging under the cuticle is also referred.

The writers emphasize the peculiar texture of the smooth muscle fibres, which only excepcionally do exist as isolated fibres, anastomosis between the fibres being the common histological appearence.

Special features of the body parenchym such as calcareous bodies and globous nuclea scattered in the parenchym are also described. 